richtsfragen an der Universität Freiburg i. Br. wurde Oberstud.-Direktor and Landtagspräsident Dr. K. Person beauftragt.

\section{Neue Fachliteratur}

Bei der Redaktion liegen folgende Fachschriften vor, deren Besprechung vorbehalten bleibt:

Commre, J. L.: Chambers' six figure mathematical tables. Vol.1: Logarithmic values. XXII u. 576 S.; Vol. 2: Natural values. XXXVI u. 576 S. W. \& R. Chambers, Edinburgh u. London 1948. Geb. pro Bd. s. 42.-.

Duscirek, A. und Hochrainer, A.: Grundzüge der Tensorrechnung in analytischer Darstellung, II. Teil: Tensoranalysis. SpringerVerlag, Wien (1950). VII u. 338 S. mit 64 Abb. Brosch. DM 24.80.

GaNs, R.: Velktoranalysis. Mit Anwendungen anf Physik und Technik. (Teubners Math. Leitfäden, Bd. 16) 7. Aufl., durchgesehen von W. STErN. B. G. Teubner Verlagsgesellschaft, Leipzig (1950). 120 S. mit 44 Abb. Kart. DM 5.90.

Grinter, L. E. (Herausgeber): Numerical methods of Analysis in engineering. Mit Beiträgen von Hardy Cross, L. E. Grinter, F.S. Shaw, R. V. Southwell, M. M. Frocht, L. M. K. Boelter, Myron Tribus, G. M. DuSinberre, Frank Baron, N. M. Newhark, Thomas J. Higgriss. The MacMillan Co., New York 1949. XVI u. 207 S. mit zahlr. Fig. Geb. $\$ 5.80$.

HAPPACH, V.: Ausgleichsrechnung. Ein Lehrbuch der Fehlerausgleichung nach der Methode der kleinsten Quadrate in Wissenschaft und Technik. (Teubners Math. Leitfäden, Bd. 18.) B. G. Teubner, Verlagsgesellschaft Leipzig 1950. 2. Aufl. 104 S. mit $26 \mathrm{Abb}$. Kart. DM 6.20.

Heffter, L.: Grundlagen und analytischer Aufbai der Projektiven, Euklidischen, Nichteuklidischen Geometrie. B. G. Teubner Verlagsgesellsch., Leipzig 1950. 2. verb. Aufl. 192 S. mit $66 \mathrm{Abb}$. Hin. DM 12.60.

Oldfindorff, F.: Die Welt der Vektoren. Einführung in Theorie und Anwendung der Vekroren, Tensoren und Operatoren. Springer-
Verlag, Wien 1950. VIII u. $470 \mathrm{~S}$. mit 68 Abb. Brosch. DM 37.50, geb. DM 40.-.

PETER, H.: Einführung in die politische Ökonomie. Verlag W. Kohlhammer, Stuttgart und Köln 1950. XVI u. 314 S. Kart. DM 15.-.

SchmeIdrer, W.: Integralgleichungen mit Anwendungen in Physik und Technik. I. Lineare Integralgleichungen (Mathematik und ihre Anwendungen in Physik und Technik, Reihe A, Bd. 22, Hrso. von E. KAMKE und A. Krazer). Akad. Verlagsgesellsch. Geest \& Portig, Leipzig 1950. XII u. $611 \mathrm{~s}$. Geb. DM 38.-.

WALc, H. S.: Analytic theory of Continued Fractions (The Universitys Series in Higher Mathematics Vol. 1; Editor M. H. Stons. Van Nostrand Comp., New York 1948, XIII u. 433 S. mit 14 Fig. Gzln $\$ 6.50$.

\section{Kurze Buchbesprechungen}

Bense, M.: Konturen einer Geistesgeschichte der Mathernatik. Verlag Classen und Goverts, Hamburg. I: Die Wathematik and die Wissenschaften, 1946, 21948, 144 S., DM 5.40. II: Die Mathematik in der Kunst, 1949, 214 S., 15. Abb. u. 3 Tf., DII 9.50 .

Unzweitelhaft üben diese beiden in der etwas eigenwilligen Sprache des temperamentvollen Autors spannend and geistreich geschriebenen Bücher mit ihrer Fülle glitzernder Gedinken auf den unbefangenen Leser starke Anziehungskraft aus. Dies bekundet sich etwa in der Tatsache, daß der erste Teil bereits in zweiter Auflage vorliegt, leider als unveränderter Nachdruck, in dem nicht einmal die vielen Druckfehler verbessert sind. Sehen wir freilich von der gefälligen änBeren Form $a b$, so müssen wir feststellen, daß sich Verf. über die Tatsachengeschichte der Mathematik, die doch den tragenden Unterbau seiner Thesen bilden müBte, nur oberflächlich und kritiklos aus längst veralteter oder als unzureichend erkannter Zweitliteratur orientiert hat. Demgemäß ist die vorliegende Darstellung nicht mehr als ein Versuch - in seiner Art beachtlich, jedoch mit unzureichenden Mitteln unternommen und daher unbefriedigend. J. E. HofmanN (Ichenhausen) 